\title{
SUPPORT VECTOR MACHINE REGRESSION FOR REACTIVITY PARAMETERS OF VINYL MONOMERS
}

\author{
XINLIANG YU*, , XUEYE WANG ${ }^{2}$ AND JIANFANG CHEN ${ }^{1}$
}

\author{
${ }^{1}$ College of Chemistry and Chemical Engineering, Hunan Institute of Engineering, Xiangtan, Hunan 411104, China \\ ${ }^{2}$ Key Laboratory of Environmentally Friendly Chemistry and Applications of Ministry of Education, College of Chemistry, Xiangtan University, \\ Xiangtan, Hunan 411105, China \\ (Received: May 5, 2010 - Accepted: May 20, 2011)
}

\begin{abstract}
Recently, the support vector machine (SVM), as a novel type of learning machine, has been introduced to solve chemical problems. In this study, $\varepsilon$ - support vector regression $(\varepsilon$-SVR) and $v$-support vector regression ( $v$-SVR) were, respectively, used to construct quantitative structure-property relationship (QSPR) models of $Q$ and $e$ parameters in the $Q-e$ scheme, which is remarkably useful in the interpretation of the reactivity of a monomer in free-radical copolymerizations. The quantum chemical descriptors used to developed the SVR models were calculated from styrene and radicals with structures $\mathrm{CH}_{3} \mathrm{CH}_{2} \mathrm{C}^{1} \mathrm{H}_{2}-\mathrm{C}^{2} \mathrm{HR}^{3} \cdot\left(\mathrm{C}^{1} \mathrm{H}_{2}=\mathrm{C}^{2} \mathrm{HR}^{3}+\right.$ $\left.\mathrm{CH}_{3} \mathrm{CH}_{2} \bullet \rightarrow \mathrm{CH}_{3} \mathrm{CH}_{2} \mathrm{C}^{1} \mathrm{H}_{2}-\mathrm{C}^{2} \mathrm{HR}^{3} \bullet\right)$. The optimum $\varepsilon$-SVR model of $\ln Q(C=9, \varepsilon=0.05$ and $\gamma=0.2)$ and the optimum $v$-SVR model of $e(C=100, v=0.5$ and $\gamma=0.4)$ produced low root mean square (rms) errors for prediction sets: 0.318 and 0.266 , respectively. Thus, applying SVR to predict parameters $Q$ and $e$ is successful.
\end{abstract}

Keywords: free-radical copolymerizations; $Q$-e scheme; quantum chemical descriptors; structure-property relations, support vector machine.

\section{INTRODUCTION}

Quantitative structure-property relationship (QSPR) studies for prediction of chemical and physical properties of molecules are unquestionably important in modern chemistry, ${ }^{1}$ especially for the cases where the reliable experimental data are difficult to obtain from experiments. Usually, a satisfactory QSPR model can serve as a guide to chemists, because it can be used to select molecules (including those not yet synthesized) with the desired properties. Thus, the QSPR approach conserves resources and accelerates the process of development of new molecules for any purpose. ${ }^{1}$

The $Q$-e scheme, as the most widely used general reactivity scheme, is remarkably useful in the interpretation of the reactivity of a monomer in freeradical copolymerizations. ${ }^{2,3}$ In the scheme, the parameter $Q$ measures the general reactivity of a monomer (or a radical) and energetic properties (i.e. thermodynamic properties); the parameter $e$ measures some polar properties of a monomer (or a radical), i.e. the supposed permanent electric charge resulting in mutual attraction or repulsion between the two monomers (or radicals). ${ }^{4}$ Many researchers have predicted the reactivity parameters $Q$ and $e$ with QSPR approaches by using the multiple linear regression (MLR) technique and/or artificial neural network (ANN) approaches. ${ }^{2,5-8}$ In fact, $Q$ and $e$ values are correlated with the reference monomer. ${ }^{2,9-11}$ While these descriptors used in these QSPR models did not include the information of the reference monomer. In the present work, support vector machine (SVM) models are developed to predict the $Q$ and $e$ values with quantum chemical descriptors obtained from radicals $\mathrm{CH}_{3} \mathrm{CH}_{2} \mathrm{C}^{1} \mathrm{H}_{2}-\mathrm{C}^{2} \mathrm{HR}^{3} \bullet\left(\mathrm{C}^{1} \mathrm{H}_{2}=\mathrm{C}^{2} \mathrm{HR}^{3}+\mathrm{CH}_{3} \mathrm{CH}_{2} \bullet \rightarrow \mathrm{CH}_{3} \mathrm{CH}_{2} \mathrm{C}^{1} \mathrm{H}_{2}-\right.$ $\mathrm{C}^{2} \mathrm{HR}^{3} \bullet$ ) and the reference monomer styrene.

\section{MATERIALS AND METHODS}

\section{Data collection}

Tables 1 and 2 show 60 vinyl monomers with double bonds and their respective experimental $\ln Q$ and $e$ values. These $\ln Q$ and $e$ values were based on the reference monomer styrene $(Q=1.0$ and $e=-0.8)$ and taken from the literature. ${ }^{12}$ These monomers comprise a variety of substitution groups, such as esters, ethers, sulfides, halides, ketones, acids, amides, aromatic and nonaromatic rings. The data were randomly split into three sets (in the ratio $50 \%, 25 \%$ and $25 \%$ ): a training set (30 monomers), a validation set (15 monomers) and a test set (15 monomers). The training set was used to train SVM models, the validation set was used to optimize the parameters of SVM models, and the prediction set was used to evaluate its prediction ability.

Table 1: Descriptors and $\ln Q$ values for 60 monomers a"

\begin{tabular}{|l|l|c|c|c|c|c|}
\hline No. & Monomer & $\begin{array}{c}E_{\beta g} / \\
\text { Hartree }\end{array}$ & $\begin{array}{c}D_{\mathrm{MC}^{2} /} \\
\text { electron }\end{array}$ & $\begin{array}{c}q_{d M R^{3} /} \\
\text { electron }\end{array}$ & exp.lnQ & $\Delta \ln Q(\mathrm{SVM})$ \\
\hline \multicolumn{2}{|l|}{ Training set } & & & & & \\
\hline 1 & Vinyl dichloroacetate & 0.22527 & 0.923259 & 0.572472 & -2.8302 & -3.0972 \\
\hline 2 & p-Vinylbenzylmethylcarbinol & 0.17202 & 0.763987 & 0.029612 & 0.5306 & 0.2694 \\
\hline 3 & Acrylate, benzyl & 0.13439 & 0.876933 & 0.433448 & -1.1087 & -0.9620 \\
\hline 4 & Acrylate, butyl & 0.16322 & 0.893779 & 0.438935 & -0.9676 & -1.0190 \\
\hline 5 & Acrylate, ethyl & 0.16385 & 0.893180 & 0.434242 & -0.8916 & -0.9632 \\
\hline 6 & Acrylate, methyl & 0.15398 & 0.881077 & 0.431427 & -0.7985 & -0.8473 \\
\hline 7 & Acrolein & 0.13006 & 0.783632 & 0.027818 & -0.2231 & -0.1717 \\
\hline 8 & Acrolein, methyl- & 0.13373 & 0.837829 & 0.247938 & 0.6043 & 0.4398 \\
\hline 9 & Acrylamide & 0.15115 & 0.913041 & 0.392726 & -1.4697 & -0.5788 \\
\hline 10 & Acrylamide, N-methylol- & 0.15173 & 0.895990 & 0.422692 & -0.6539 & -0.8343 \\
\hline 11 & Acrylonitrile & 0.21346 & 0.859496 & 0.170140 & -0.7340 & -0.7826 \\
\hline
\end{tabular}




\begin{tabular}{|c|c|c|c|c|c|c|}
\hline 12 & Acryloyl chloride & 0.15213 & 0.828820 & 0.106172 & 0.5988 & 0.5482 \\
\hline 13 & Allyl acetate & 0.22083 & 1.020450 & 0.233563 & -1.4271 & -1.4773 \\
\hline 14 & Allyl chloride & 0.24387 & 1.031016 & 0.559691 & -3.6497 & -3.7004 \\
\hline 15 & Butadiene & 0.21354 & 0.656558 & 0.236536 & 0.5306 & 0.4802 \\
\hline 16 & Butadiene-1-carboxylate, ethyl & 0.15988 & 0.618823 & 0.268439 & 0.5128 & 0.4631 \\
\hline 17 & Butadiene, 2-fluoro- & 0.18836 & 0.662607 & 0.258051 & 0.6313 & 0.6078 \\
\hline 18 & Imidazole, 1-vinyl- & 0.16365 & 0.863881 & 0.553052 & -2.2073 & -2.1570 \\
\hline 19 & Oxazolidone, N-vinyl- & 0.23862 & 0.869334 & 0.620975 & -2.4418 & -2.5362 \\
\hline 20 & Propylene & 0.29195 & 1.024493 & 0.646354 & -4.7105 & -4.6616 \\
\hline 21 & Pyridine, 2-methyl-5-vinyl- & 0.16936 & 0.761578 & 0.007327 & 0.2776 & 0.2272 \\
\hline 22 & Pyridine, 2-vinyl- & 0.16393 & 0.777410 & 0.131296 & 0.3436 & 0.3948 \\
\hline 23 & Pyridine, 2-vinyl-5-ethyl- & 0.16408 & 0.770506 & 0.132219 & 0.2546 & 0.3760 \\
\hline 24 & Quinoline, 2-vinyl- & 0.13819 & 0.755183 & 0.146547 & 0.0392 & 0.0732 \\
\hline 25 & Vinyl benzoate & 0.19670 & 0.903608 & 0.626195 & -3.5066 & -3.2253 \\
\hline 26 & Vinyl bromide & 0.22064 & 0.955858 & 0.273547 & -3.2702 & -1.7856 \\
\hline 27 & Vinyl butyl ether & 0.27566 & 0.880772 & 0.617282 & -3.2702 & -3.2201 \\
\hline 28 & Vinyl butyrate & 0.23046 & 0.924082 & 0.606284 & -3.7297 & -3.3402 \\
\hline 29 & Vinyl chloride & 0.24777 & 0.956987 & 0.195952 & -2.8824 & -2.8336 \\
\hline 30 & Vinyl chloroacetate & 0.21772 & 0.933369 & 0.589633 & -3.2442 & -3.2597 \\
\hline \multicolumn{7}{|c|}{ Validation set } \\
\hline 31 & Vinyl formate & 0.23388 & 0.922994 & 0.550924 & -3.1466 & -3.1008 \\
\hline 32 & Vinylisocyanate & 0.23332 & 0.858007 & 0.565474 & -1.9661 & -2.0701 \\
\hline 33 & Vinyltrimethylsilane & 0.22412 & 0.981151 & 0.518180 & -3.6119 & -3.1643 \\
\hline 34 & Styrene & 0.17306 & 0.771092 & 0.025964 & 0.0000 & 0.2821 \\
\hline 35 & Styrene, m-bromo- & 0.15657 & 0.769569 & 0.023489 & 0.2231 & 0.1515 \\
\hline 36 & Styrene, p-bromo- & 0.16878 & 0.758891 & 0.030488 & 0.2624 & 0.2418 \\
\hline 37 & Vinyl hendecanoate & 0.23409 & 0.935626 & 0.619110 & -2.8824 & -3.5734 \\
\hline 38 & Vinyl isobutyl ether & 0.27411 & 0.878907 & 0.612383 & -3.5066 & -3.1587 \\
\hline 39 & Vinyl isobutyl sulfide & 0.21112 & 0.867871 & 0.022656 & -0.7133 & -0.5602 \\
\hline 40 & Styrene, p-methyl- & 0.17450 & 0.764417 & 0.027010 & 0.0953 & 0.2771 \\
\hline 41 & Styrene, p-1-(2-hydroxybutyl)- & 0.17440 & 0.753247 & 0.025863 & -0.3567 & 0.2611 \\
\hline 42 & Styrene, p-2-(2-hydroxypropyl)- & 0.17291 & 0.748369 & 0.032011 & 0.1398 & 0.2518 \\
\hline 43 & Acrylic acid & 0.16414 & 0.898319 & 0.386303 & -0.1863 & -0.4888 \\
\hline 44 & Vinyl phenyl ketone & 0.13210 & 0.821919 & 0.194540 & 0.1484 & 0.4236 \\
\hline 45 & Allyl acrylate & 0.21037 & 1.003116 & 0.260128 & -1.1394 & -1.2117 \\
\hline \multicolumn{7}{|c|}{ Test set } \\
\hline 46 & Acrylate, glycidyl & 0.16195 & 0.888131 & 0.444600 & -0.7340 & -1.0432 \\
\hline 47 & Vinyl dodecyl ether & 0.27545 & 0.880658 & 0.617658 & -3.1942 & -3.2144 \\
\hline 48 & Vinyl Ethyl oxalate & 0.19077 & 0.888536 & 0.579208 & -2.8824 & -2.5940 \\
\hline 49 & Vinyl octyl ether & 0.27549 & 0.880666 & 0.617633 & -3.9120 & -3.2153 \\
\hline 50 & Styrene, p-chloro- & 0.17469 & 0.759552 & 0.028157 & 0.2852 & 0.2723 \\
\hline 51 & Styrene, p-chloromethyl- & 0.16888 & 0.749743 & 0.028387 & 0.3293 & 0.2214 \\
\hline 52 & Styrene, p-methoxy- & 0.16942 & 0.759190 & 0.033771 & 0.4253 & 0.2503 \\
\hline 53 & Vinyl isothiocyanate & 0.16329 & 0.753847 & 0.448005 & -0.5276 & -0.1506 \\
\hline 54 & Vinyl methyl sulfide & 0.21496 & 0.875676 & 0.016800 & -0.8675 & -0.7290 \\
\hline 55 & N-Vinylcaprolactam & 0.20846 & 0.853726 & 0.583579 & -1.9661 & -2.0242 \\
\hline 56 & Vinyl pelargonate & 0.23192 & 0.926946 & 0.619105 & -3.0791 & -3.4516 \\
\hline 57 & Acrylate, heptafluorobutyl & 0.16489 & 0.848580 & 0.442982 & -0.0408 & -0.7361 \\
\hline 58 & Vinyl propionate & 0.22507 & 0.938147 & 0.619306 & -3.6119 & -3.5441 \\
\hline 59 & Vinyl acetate & 0.23176 & 0.921440 & 0.603176 & -3.6497 & -3.2997 \\
\hline 60 & Vinyl N,N-diethylcarbamate & 0.21120 & 0.929291 & 0.653208 & -3.5756 & -3.6700 \\
\hline
\end{tabular}

${ }^{\text {a }}$ The unit of $E_{\beta g}: 1$ Hartree $=2.6255 \times 10^{6} \mathrm{~J} / \mathrm{mol}$; the unit of $D_{\mathrm{MC}^{2}}$ and $q_{d M R^{3}}: 1$ electron $=1.602188 \times 10^{-19} \mathrm{C}$; 
Table 2. Descriptors and $e$ values for 60 monomers ${ }^{\mathrm{a}}$

\begin{tabular}{|c|c|c|c|c|c|}
\hline No. & Monomer & $\begin{array}{c}E_{\alpha \mathrm{g}} / \\
\text { Hartree }\end{array}$ & $\begin{array}{c}e_{\mathrm{g}}{ }_{\mathrm{g}} \\
\text { Hartree }\end{array}$ & exp. $\ln Q$ & $\begin{array}{c}\Delta \ln Q \\
(\mathrm{SVM})\end{array}$ \\
\hline \multicolumn{6}{|c|}{ Training set } \\
\hline 1 & Vinyl dichloroacetate & 0.14756 & 0.15183 & -1.38 & -1.03 \\
\hline 2 & p-Vinylbenzylmethylcarbinol & 0.17070 & 0.15764 & -1.27 & -1.12 \\
\hline 3 & Acrylate, benzyl & 0.22966 & 0.11062 & 1.13 & 1.39 \\
\hline 4 & Acrylate, butyl & 0.27810 & 0.12268 & 0.85 & 0.77 \\
\hline 5 & Acrylate, ethyl & 0.27835 & 0.12138 & 0.55 & 0.78 \\
\hline 6 & Acrylate, methyl & 0.28146 & 0.11256 & 0.64 & 0.90 \\
\hline 7 & Acrolein & 0.25861 & 0.10660 & 1.31 & 1.27 \\
\hline 8 & Acrolein, methyl- & 0.25274 & 0.11601 & 0.71 & 1.11 \\
\hline 9 & Acrylamide & 0.27807 & 0.12988 & 0.54 & 0.68 \\
\hline 10 & Acrylamide, N-methylol- & 0.26761 & 0.11943 & 1.15 & 0.91 \\
\hline 11 & Acrylonitrile & 0.25874 & 0.11439 & 1.23 & 1.09 \\
\hline 12 & Acryloyl chloride & 0.26002 & 0.08420 & 1.92 & 1.77 \\
\hline 13 & Allyl acetate & 0.21165 & 0.17571 & -1.07 & -0.89 \\
\hline 14 & Allyl chloride & 0.22177 & 0.17430 & -0.6 & -0.75 \\
\hline 15 & Butadiene & 0.23326 & 0.16208 & -0.5 & -0.15 \\
\hline 16 & Butadiene-1-carboxylate, ethyl & 0.20552 & 0.12074 & 1.26 & 1.11 \\
\hline 17 & Butadiene, 2-fluoro- & 0.25123 & 0.14804 & 0.63 & 0.48 \\
\hline 18 & Imidazole, 1-vinyl- & 0.21430 & 0.16160 & -0.68 & -0.42 \\
\hline 19 & Oxazolidone, N-vinyl- & 0.20854 & 0.18589 & -1.7 & -1.09 \\
\hline 20 & Propylene & 0.27730 & 0.19467 & -1.69 & -1.43 \\
\hline 21 & Pyridine, 2-methyl-5-vinyl- & 0.15595 & 0.14634 & -0.66 & -0.81 \\
\hline 22 & Pyridine, 2-vinyl- & 0.16770 & 0.14072 & -0.42 & -0.40 \\
\hline 23 & Pyridine, 2-vinyl-5-ethyl- & 0.16354 & 0.14440 & -0.91 & -0.65 \\
\hline 24 & Quinoline, 2-vinyl- & 0.14086 & 0.13472 & -0.09 & -0.24 \\
\hline 25 & Vinyl benzoate & 0.13120 & 0.16024 & -0.89 & -1.04 \\
\hline 26 & Vinyl bromide & 0.21676 & 0.16365 & -0.23 & -0.45 \\
\hline 27 & Vinyl butyl ether & 0.24294 & 0.21005 & -1.5 & -1.46 \\
\hline 28 & Vinyl butyrate & 0.18616 & 0.17756 & -0.89 & -1.16 \\
\hline 29 & Vinyl chloride & 0.24043 & 0.16753 & 0.16 & -0.32 \\
\hline 30 & Vinyl chloroacetate & 0.16351 & 0.15853 & -1.61 & -1.20 \\
\hline \multicolumn{6}{|c|}{ Validation set } \\
\hline 31 & Vinyl formate & 0.18889 & 0.16830 & -1.19 & -1.07 \\
\hline 32 & Vinylisocyanate & 0.20546 & 0.16112 & -0.95 & -0.58 \\
\hline 33 & Vinyltrimethylsilane & 0.27045 & 0.16390 & 0.19 & -0.10 \\
\hline 34 & Styrene & 0.17321 & 0.15264 & -0.8 & -0.92 \\
\hline 35 & Styrene, m-bromo- & 0.17065 & 0.14028 & -0.27 & -0.34 \\
\hline 36 & Styrene, p-bromo- & 0.16577 & 0.14181 & -0.68 & -0.49 \\
\hline 37 & Vinyl hendecanoate & 0.18359 & 0.18347 & -0.84 & -1.14 \\
\hline 38 & Vinyl isobutyl ether & 0.23721 & 0.21144 & -1.27 & -1.39 \\
\hline 39 & Vinyl isobutyl sulfide & 0.20120 & 0.18655 & -0.95 & -1.10 \\
\hline 40 & Styrene, p-methyl- & 0.16921 & 0.15609 & -0.63 & -1.09 \\
\hline 41 & Styrene, p-1-(2-hydroxybutyl)- & 0.16461 & 0.14812 & -0.97 & -0.83 \\
\hline 42 & Styrene, p-2-(2-hydroxypropyl)- & 0.16770 & 0.15161 & -0.49 & -0.95 \\
\hline 43 & Acrylic acid & 0.28017 & 0.11669 & 0.88 & 0.83 \\
\hline 44 & Vinyl phenyl ketone & 0.19062 & 0.11140 & 1.02 & 1.32 \\
\hline 45 & Allyl acrylate & 0.15893 & 0.16424 & -0.99 & -1.30 \\
\hline \multicolumn{6}{|c|}{ Test set } \\
\hline 46 & Acrylate, glycidyl & 0.27627 & 0.11621 & 1.28 & 0.88 \\
\hline 47 & Vinyl dodecyl ether & 0.23920 & 0.21037 & -1.69 & -1.42 \\
\hline 48 & Vinyl Ethyl oxalate & 0.13021 & 0.14588 & -0.65 & -0.69 \\
\hline 49 & Vinyl octyl ether & 0.23976 & 0.21032 & -1.57 & -1.43 \\
\hline 50 & Styrene, p-chloro- & 0.16572 & 0.14240 & -0.64 & -0.52 \\
\hline 51 & Styrene, p-chloromethyl- & 0.16803 & 0.13749 & -0.38 & -0.21 \\
\hline 52 & Styrene, p-methoxy- & 0.15622 & 0.16310 & -1.4 & -1.29 \\
\hline
\end{tabular}




\begin{tabular}{|l|l|l|l|l|l|}
\hline 53 & Vinyl isothiocyanate & 0.18356 & 0.13471 & 0.37 & 0.19 \\
\hline 54 & Vinyl methyl sulfide & 0.19857 & 0.18799 & -1.66 & -1.11 \\
\hline 55 & N-Vinylcaprolactam & 0.17236 & 0.19400 & -1.18 & -0.93 \\
\hline 56 & Vinyl pelargonate & 0.18918 & 0.18037 & -1.22 & -1.14 \\
\hline 57 & Acrylate, heptafluorobutyl & 0.26690 & 0.08765 & 1.34 & 1.66 \\
\hline 58 & Vinyl propionate & 0.19665 & 0.17664 & -0.68 & -1.06 \\
\hline 59 & Vinyl acetate & 0.18766 & 0.17801 & -0.88 & -1.15 \\
\hline 60 & Vinyl N,N-diethylcarbamate & 0.22595 & 0.19458 & -1.1 & -1.25 \\
\hline
\end{tabular}

${ }^{\text {a }}$ The unit of $E_{\text {ag }}$ and $e_{\mathrm{g}}: 1$ Hartree $=2.6255 \times 10^{6} \mathrm{~J} / \mathrm{mol}$.

\section{Molecular descriptors}

Previous works have found that atomic charges and frontier molecular orbital energies are related to $\ln O$ and $e$. $^{2,6-9}$ Thus, these descriptors were calculated for radicals $\mathrm{CH}_{3} \mathrm{CH}_{2} \mathrm{C}^{1} \mathrm{H}_{2}-\mathrm{C}^{2} \mathrm{HR}^{3}$ • with density functional theory (DFT) in Gaussian $03^{13}$ program, at the UB3LYP level of theory with 6-31G(d) basis set. Calculations were also performed on styrene using the same methods. Descriptors calculated from radicals include the energies of the highest occupied molecular orbital (HOMO) and the lowest unoccupied molecular orbital (LUMO) of alpha spin states $\left(E_{\text {aномо }}\right.$ and $\left.E_{\text {aLUMO }}\right)$, the energies of HOMO and LUMO of beta spin states $\left(E_{\text {вномо }}\right.$ and $\left.E_{\text {вLUMO }}\right)$, the energy gap between HOMO and LUMO of alpha spin states $\left(E_{\alpha \mathrm{g}}\right)$ and the energy gap between HOMO and LUMO of beta spin $\left(E_{\text {p }}\right)$, Mulliken charges of $\mathrm{R}^{3}\left(q_{\mathrm{MR}^{3}}\right)$, and Mulliken atomic spin densities on $\mathrm{C}^{2}\left(D_{\mathrm{MC}^{2}}\right)$. The descriptors for styrene were Mulliken atomic charges of $\mathrm{R}^{3}\left(q_{\mathrm{SRR}^{3}}\right)$ and the HOMO energy $\left(E_{\text {sномо }}\right)$. In addition, we defined two descriptors $q_{\mathrm{DMR}^{3}}$ and $e_{\mathrm{g}}$. The former was equal to the absolute value of the Mulliken charge difference on $\mathrm{R}^{3}$ between radicals and styrene, i.e., $q_{\mathrm{DMR}^{3}}=\operatorname{abs}\left(q_{\mathrm{MR}^{3}} q_{\mathrm{SMR}^{3}}\right)$. The latter is the absolute value of the energy difference between the LUMO energy of beta spin states $\left(E_{\text {BLUMO }}\right)$ of radicals and the HOMO energy of styrene. it can be expressed as: $e_{\mathrm{g}}$ $=\operatorname{abs}\left(E_{\text {вLuмо }}-E_{\text {sномо }}\right)$. A total of 12 descriptors were calculated.

\section{Support vector machine}

Support vector machines (SVM) ${ }^{14-25}$ are a powerful state-of-the-art data mining algorithm for nonlinear input-output knowledge discovery. In SVM, the idea is to map the input data into a high dimensional feature space and subsequently carry out the linear regression in the feature space. ${ }^{14,15}$ Thus, the input-output pairs of training data of size $n$

$\left(x_{1}, y_{1}\right)\left(x_{2}, y_{2}\right), \ldots,\left(x_{p}, y_{1}\right) x_{i} \in R^{n}, y_{i} \in R(i=1,2, \ldots, n)$

can be expressed as

$$
f(\mathrm{x})=\sum_{i}^{n} \phi\left(\mathrm{x}_{i}\right) \omega+b
$$

where $\mathbf{x}_{\text {i }}$ is an input example, $\Phi$ is a nonlinear mapping, $w$ is a weight vector and $b$ is the bias of the regression function. ${ }^{15,16} \mathrm{~A}$ loss function called $\varepsilon$-insensitive loss had been proposed in the context of the $\varepsilon$-insensitive support vector regression $\left(\varepsilon\right.$-SVR). ${ }^{15,16}$

$$
C(y, f(\mathbf{x}, \omega))=\left\{\begin{array}{cc}
0 & \text { if }|y-f(\mathbf{x}, \omega)| \leq \varepsilon \\
|y-f(\mathbf{x}, \omega)|-\varepsilon & \text { otherwise }
\end{array}\right.
$$

The standard SVR amounts to (simultaneous) minimization of the norm of the squared weight vector, $\|\mathrm{w}\|^{2}$, and minimization of the sum of permitted errors. To measure the deviation of training samples outside $\boldsymbol{E}_{\text {-insensitive }}$ zone, non-negative slack variables $\xi_{i}$ and $\xi_{i}^{*}(i=1,2, \ldots, n)$ are introduced. Thus, $\varepsilon$-SVR can be formulated as minimization of the following functional

$$
\min _{w, b, \xi, \xi^{*}} J\left(w, \xi, \xi^{*}, b\right)=\frac{1}{2}\|w\|^{2}+C \sum_{i}^{n}\left(\xi_{i}+\xi_{i}^{*}\right)
$$

subject to:

$$
\begin{aligned}
& \phi^{T}\left(x_{i}\right) w+b-y_{i} \leq \varepsilon+\xi_{i} \\
& y_{i}-\phi^{T}\left(x_{i}\right) w-b \leq \varepsilon+\xi_{i}^{*} \\
& \xi_{i}, \xi_{i}^{*} \geq 0 \quad i=1,2, \ldots, n
\end{aligned}
$$

where $\varepsilon$ is a prescribed parameter of the $\varepsilon$-insensitive loss function and $C$ is a penalization parameter. This optimization problem can be transformed into the dual problem and the final solution can be expressed as

$$
f(\mathrm{x})=\sum_{i}^{n_{\mathrm{v}}}\left(a_{i}-a_{i}^{*}\right) \phi\left(\mathrm{x}_{i}\right) \cdot \phi(\mathrm{x})+b
$$

where the coefficients $\alpha_{i}$ and $\alpha_{i}^{*}$ are in the ranges of $0 \leq \alpha_{i}, \alpha_{i}^{*} \leq \mathrm{C}$, respectively, and $n_{\mathrm{sv}}$ is the number of support vectors. In general, $n_{\mathrm{sv}}$ is much smaller than the number of training samples since only a fraction of training samples appear with non-zero coefficients in Eq. 7. These training samples (vectors) are known as the support vectors and hence the name SVM.

According to the SVM training methodology, ${ }^{15,16}$ one does not need to know explicitly the form of the mapping $\Phi(\mathrm{x})$, but only a kernel function, which maps the samples into a high dimensional space. When a kernel satisfies certain requirements (Mercer's theorem), it can be defined connecting the dot products of the feature vectors to the vectors in the input space

$$
K\left(\mathrm{x}, \mathrm{x}_{i}\right)=\phi\left(\mathrm{x}_{i}\right) \cdot \phi(\mathrm{x})
$$

The SVM models used in this study implement the Gaussian (or radial basis function, RBF) kernel, given by:

$$
K\left(\mathrm{x}_{i}, \mathrm{x}_{j}\right)=\exp \left(-\gamma\left\|\mathrm{x}_{i}-\mathrm{x}_{j}\right\|^{2}\right)
$$

where $\gamma$ is a parameter to be optimized. $\gamma$ controls the amplitude of the Gaussian function and, therefore, controls the generalization ability of SVM. The RBF function is one of the most commonly used kernel function in the SVR technique and has been used widely in SVM. ${ }^{15,16}$

Besides the parameter $\gamma$, the parameters $C$ and $\varepsilon$ also need to be adjusted by users when a $\varepsilon$-SVR model is trained. The parameter $C$, the penalty factor, controls the trade off between errors of the SVM on training data and model complexity. The parameter $\varepsilon$ controls the width of the $\varepsilon$-insensitive zone and determines the complexity and the generalization capability of the network.

The tube parameter $\varepsilon$ is difficult to select as one does not know beforehand how accurately the function will fit. The $v$-SVR was developed to automatically adjust the tube size, $\varepsilon$, by using a parameter $v$. In the $v$-SVR, the parameter $v$ replaces the parameter $\varepsilon$ of the $\varepsilon$-SVR and used to control the number of support vectors. ${ }^{24,25}$ Similar to Eq. 3 , the primal form is 


$$
\min _{w, b, \xi, \xi^{*}, \varepsilon} J\left(w, \xi, \xi^{*}, b\right)=\frac{1}{2}\|w\|^{2}+C v \varepsilon+\frac{C}{n} \sum_{i}^{n}\left(\xi_{i}+\xi_{i}^{*}\right)
$$

subject to:

$$
\begin{aligned}
& \phi^{T}\left(x_{i}\right) w+b-y_{i} \leq \varepsilon+\xi_{i} \\
& y_{i}-\phi^{T}\left(x_{i}\right) w-b \leq \varepsilon+\xi_{i}^{*} \\
& \xi_{i}, \xi_{i}^{*} \geq 0 \quad i=1,2, \ldots, n, \quad \varepsilon \geq 0
\end{aligned}
$$

The final function, , is the same as that of the $\varepsilon$-SVR (see Eq.7). When RBF kernel is still adopted, three parameters $C, v$ and $\gamma$ should be tuned in $v$-SVR models.

All SVM models from the present paper were obtained with winSVM, which is freely available for download (http://www.cs.ucl.ac.uk/staff/M. Sewell/winsvm/). Stepwise multiple linear regression (MLR) was used to select an optimum subset of descriptors and develop a MLR model. Then these descriptors were used to develop SVM models.

\section{RESULTS AND DISCUSSION}

By carrying out the correlation between the 12 descriptors and reactivity parameters $\ln Q$ and $e$ in the training sets with stepwise MLR, the optimal MLR models of $\ln Q$ and $e$ were obtained. The optimum subset of descriptors in $\ln Q$ comprises three descriptors: $E_{\beta \mathrm{g}}$ (the energy gap between HOMO and LUMO of beta spin), $D_{\mathrm{MC}^{2}}$ (Mulliken atomic spin densities on $\mathrm{C}^{2}$ ), and $q_{d M R^{3}}$ (the absolute value of the Mulliken charge difference on $\mathrm{R}^{3}$ between radical and styrene). Statistical parameters corresponding to the MLR model are the following:

$$
\begin{aligned}
& \ln Q=7.804-13.537 E_{\beta \mathrm{g}}-6.433 D_{M C^{2}}-3.011 q_{d M R^{3}} \\
& R=0.940, R^{2}=0.883, n=30, s e=0.596, F=65.682
\end{aligned}
$$

where $N$ is the number of monomers used, $R$ is the correlation coefficient, $s e$ is the standard error of estimation, $F$ is the Fischer's ratio. The the root mean square (rms) errors for the training, validation and prediction sets are 0.555 , 0.578 and 0.496 , respectively.

By the view of the frontier molecular orbital (FMO) theory of chemical reactivity, the formation of a transition state is due to the HOMO (electron-rich component) and LUMO (electron-deficient component) interaction. Thus, the FMOs are separated from the other orbitals and become very popular quantum chemical descriptors. In general, the HOMO energy can describe ionization potential; while the LUMO energy can reflect the electron affinity. ${ }^{1}$ Moreover, both the HOMO and the LUMO energies are important in radical reactions. ${ }^{26} E_{\text {g }}$ (the energy gap between HOMO and LUMO) is an important stability index. For example, a large $E_{\mathrm{g}}$ value implies high stability for the molecule in the sense of its lower reactivity in chemical reactions. ${ }^{27,28}$ The parameter $Q$ measures of the resonance stabilization, i.e. a monomer, that form free radicals easily, possesses a large $\ln Q$ value. Thus, it is easy to understand that a radical with a small $E_{\beta \mathrm{g}}$ value would have high reactivity and a $\operatorname{large} \ln Q$ value.

In fact, all chemical interactions are resulted from electrostatic (or orbital), which are based on atomic charges. ${ }^{1}$ Atomic charge descriptors can reflect molecular chemical reactivity (or intermolecular interactions). A large $D_{\mathrm{MC}^{2}}$ or $q_{d \mathrm{MR}^{3}}$ implies that the monomer (or the radical) possesses a small resonance stabilization and has a small $\ln Q$ value.

The optimum MLR model of the reactivity parameter $e$ includes two descriptors, $E_{a \mathrm{~g}}$ and $e_{\mathrm{g}}$. Statistical parameters for $e$ are the following

$\mathrm{e}=2.339+7.314 E_{\mathrm{ag}}-27.988 e_{\mathrm{g}}$

$R=0.925, R^{2}=0.856, n=30, \mathrm{ge}=0.417, F=80.431$

The rms errors for the training, validation and prediction sets are 0.395 , 0.337 and 0.343 , respectively. The parameter $e$ is a measure of the polarity of a monomer (or a radical). In Eq. 15, the two descriptors $E_{\text {ag }}$ and $e_{\mathrm{g}}$ are related to the FMO energies of radicals. Usually, the FMO energies (or the energy gap $E_{\mathrm{g}}$ between HOMO and LUMO) are correlated with the polarization of a molecule. ${ }^{1}$ Thus, $E_{\alpha g}$ and $e_{\mathrm{g}}$ are also related to the reactivity parameter $e$.

The program winSVM was used to develop SVM models for $\ln Q$ and $e$. In order to get a satisfactory model, SVM parameters $C, \varepsilon$ (or $v$ ) and $\gamma$ need to be selected properly. Here, take the training of SVM models of $\ln Q$ as an example. Firstly, the training set of $\ln Q$ was selected as the input file and optimized 100 times. Then the output results were inspected. Learning parameters of $C=10$, $\varepsilon=0.01$ and $\gamma=0.2$ produced a low mean squared error. Thus, these SVM parameters were used for the validation set and optimized furtherly. By training the SVM models of $\ln Q$ with different parameters $\gamma$ of $0.1,0.15,0.2,0.25,0.3$ and $0.4(C=10, \varepsilon=0.01)$, the rms errors of the validation set are $0.384,0.353$, $0.332,0.353,0.372$ and 0.443 , respectively. Thus, the optimal $\gamma$ corresponding to the minimal rms error was set to 0.2 . Subsequently, by using $\gamma=0.2$ and $C$ $=10$, another parameter $\varepsilon$ was optimized with $\varepsilon$ being $0.005,0.01,0.03,0.05$, 0.07 and 0.08 , respectively. The validation set rms errors based on different $\varepsilon$ are $0.334,0.332,0.323,0.319,0.320,0.330$ and 0.327 , respectively, which shows that the optimal $\varepsilon$ was fixed to 0.05 . Similarly, the last parameter $C$ based on $\varepsilon$ of 0.05 and $\gamma$ of 0.2 was optimized. The validation set rms errors are $0.337,0.320,0.318,0.319,0.322$ and 0.326 , respectively, when the parameter $C$ was tuned with $C$ of $5,8,9,10,11$ and 12 . Thus, the optimal $C$ was 9. Lastly, the optimum $\varepsilon$-SVR model of $\ln Q$ with the RBF kernel $(C=9, \varepsilon=0.05$ and $\gamma=$ 0.2 ) was tested by the prediction set. The rms errors for the training, validation and prediction sets are $0.343,0.330$ and 0.317 , respectively. The $\ln Q$ values calculated with the $\varepsilon$-SVR model are listed in Table 1 and depicted in Figure 1.

The SVM parameters of $e$ were tuned with the same methods. Learning parameters of $C=100, v=0.6$ and $\gamma=0.6$ were obtained after initial optimization. Then the different parameters $\gamma(0.3,0.4,0.5,0.6,0.7$, and 0.8$), v(0.2,0.3,0.4$, $0.5,0.6$ and 0.7$)$, and $C(10,50,90,100,110,130)$ were tested. In the end, the optimal SVM parameters $(\gamma=0.4, v=0.5$ and $C=100)$ were obtained. The optimal $v$-SVR model produced rms errors of 0.257 for the training set, 0.264 for the validation set and 0.266 for the prediction set. The calculated $e$ values from the $v$-SVR model are listed in Table 2 and depicted in Figure 2.

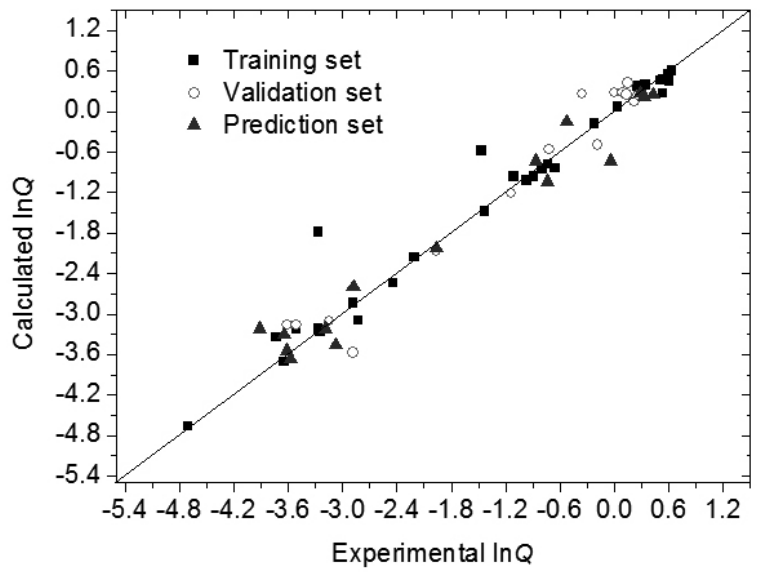

Figure 1. Plot of experimental $\ln Q$ versus calculated $\ln Q$ with the $\varepsilon$-SVR model.

The rms errors for prediction sets of the $\ln Q$ and $e$ models based on the ANN approach were 0.313 and 0.271 , respectively [8], which are close to the results obtained with the SVM approach. But the ratio (30/30) of fitted samples $(15+15=30)$ to training samples $(30)$ in this paper are larger than that $(16 / 40)$ in previous model [8]. This means that the present SVM models have better statistical quality and generalization capability. For the previous ANN models of vinyl monomers, the training set rms errors were 0.581 for $\ln Q$ and 0.234 for $e .{ }^{6}$ In addition, in the ANN models of acrylate monomers, the training set rms errors were 0.302 for $\ln Q$ and 0.127 for $e .^{7}$ In fact, as long as the correlation coefficient $R$ between the experimental and calculated $e$ values is greater than 0.876 , then a good fit has been achieved. ${ }^{2}$ The $R$ values of the $v$-SVR model for $e$ in this paper are 0.971 for the training set, 0.943 for the validation set and 0.960 for the prediction set, which are larger than 0.876 . This illustrates that our results for the model $e$ are satisfactory and acceptable. In comparison with previous other models, the present SVM model shows satisfying statistical results, although the number of samples used in this article is much greater than that in previous models. ${ }^{2,5-7}$ 


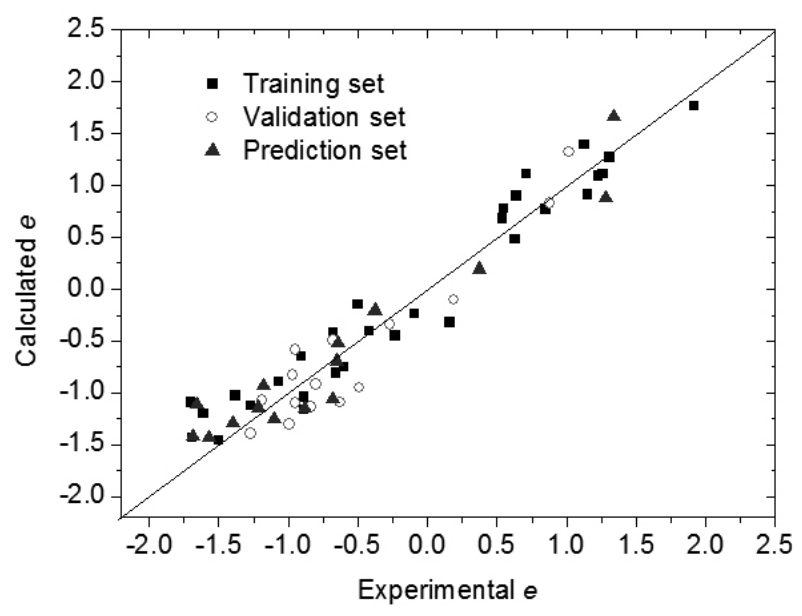

Figure 2. Plot of experimental $e$ versus calculated $e$ with the $v$-SVR model.

\section{CONCLUSIONS}

Two SVR models were developed to predict the reactivity parameters $Q$ and $e$, respectively, of vinyl monomers in radical copolymerization. Comparison to existing models, the SVM models shows good statistical characteristics. We have the following conclusions:

1) To developed the SVR models, calculating quantum chemical descriptors from styrene and radicals with structures $\mathrm{CH}_{3} \mathrm{CH}_{2} \mathrm{C}^{1} \mathrm{H}_{2}-\mathrm{C}^{2} \mathrm{HR}^{3}$ • formed from $\mathrm{C}^{1} \mathrm{H}_{2}=\mathrm{C}^{2} \mathrm{HR}^{3}$ and $\mathrm{CH}_{3} \mathrm{CH}_{2} \bullet$ is feasible.

2) The SVR models describing the non-linear correlation between the quantum chemical descriptors and reactivity parameters $Q$ and $e$ is accurate and acceptable.

3) Atom charges ( $D_{\mathrm{MC}^{2}}$ and $q_{d \mathrm{MR}^{3}}$ ) and frontier molecular orbital energies $\left(E_{\beta \mathrm{g}}, E_{\alpha \mathrm{g}}\right.$ and $e_{\mathrm{g}}$ ) are the most important factors in predicting monomer (or radical) reactivity based on the reference monomer styrene $(Q=1.0$ and $e=$ $-0.8)$.

\section{ACKNOWLEDGEMENTS}

We thank the financial supports from the Open Project Program of Key Laboratory of Environmentally Friendly Chemistry and Applications of Ministry of Education, China (Grant No. 10HJYH06), Scientific Research Fund of Hunan Provincial Education Department (Grant No. 09A019), and the National Natural Science Foundation of China (Grant No. 20972045).

\section{REFERENCES}

1. M. Karelson, V. S. Lobanov, A. R. Katritzky, Chem. Rev. 96, 1027, (1996).

2. C. G. Zhan, D. A. Dixon, J. Phys. Chem. A, 106, 10311, (2002).

3. A. D. Jenkins, J. Polym. Sci. A: Polym. Chem., 37, 113, (1999).

4. T. Alfrey, C. C. Price, J. Polym. Sci. 2, 101, (1947).

5. A. A. Toropov, V. O. Kudyshkin, N. L. Voropaeva, I. N. Ruban, S. Sh. Rashidova, J. Struct. Chem. 45, 945, (2004).

6. X. L. Yu, W. Q. Liu, F. Liu, X. Y. Wang, J. Mol. Model. 14, 1065, (2008).

7. X. L. Yu, B. Yi, X. Y. Wang, Eur. Polym. J. 44, 3997, (2008).

8. X. L. Yu, X. Y. Wang, B. Li, Colloid. Polym. Sci. 288, 951, (2010).

9. S. C. Rogers, W. C. Mackrodt, T. P .Davis, Polymer 35, 1258, (1994)

10. G. C. Laurier, K. F. O'driscoll, P. M. Reilly, J. Polym. Sci.: Polym. Symp. Ed. 72, 17, (1985).

11. N. Kawabata, T. Tsuruta, J. Furukawa, Makromol. Chem. 51, 70, (1962).

12. J. Brandrup, E. H. Immergut, E. A. Grulke, Polymer Handbook, 4th ed. Wiley, New York, 1999.

13. M. J. Frisch, G.W. Trucks, H. B. Schlegel, G. E. Scuseria, M. A. Robb, J. R. Cheeseman, V. G. Zakrzewski, J. A. Montgomery, R. E. Stratmann, J. C. Burant, S. Dapprich, J. M. Millam, A. D. Daniels, K. N. Kudin, M. C. Strain, O. Farkas, J. Tomasi, V. Barone, M. Cossi, R. Cammi, B. Mennucci, C. Pomelli, C. Adamo, S. Clifford, J. Ochterski, G. A. Petersson, P. Y. Ayala, Q. Cui, K. Morokuma, D. K. Malick, A. D. Rabuck, K. Raghavachari, J. B. Foresman, J. Cioslowski, J. V. Ortiz, B. B. Stefanov,
G. Liu, A. Liashenko, P. Piskorz, I. Komaromi, R. Gomperts, R. L. Martin, D. J. Fox, T. Keith, M. A. Al-Laham, Peng, W. Chen, M. W. Wong, J. L. Andres, M. Head-Gordon, E. S. Replogle, and J. A. Pople, Gaussian 03, Revision B.05. Gaussian Inc., Pittsburgh, PA, 2003.

14. V. Vapnik, The Nature of Statistical Learning Theory. New York, Springer, 1995.

15. V. N. Vapnik, Statistical Learning Theory. Wiley, New York, 1998.

16. G. Camps-Valls, A. M. Chalk, A. J. Serrano-López, J.D. Martín-Guerrero, E. L. Sonnhammer, BMC Bioinformatics, 5, 135, (2004).

17. R. Kumar, A. Kulkarni, V. K. Jayaraman, B. D. Kulkarni, Internet Electron J. Mol. Des., 3, 118, (2004).

18. C. J. C. Burges, Data Min. Knowl. Disc., 2, 121, (1998).

19. F. Luan, R. Zhang, X. Yao, M. Liu, Z. Hu, B. Fan, QSAR Comb. Sci., 24, 227, (2005).

20. H. D. Li, Y. Z. Liang, Q. S. Xu, Chemomet. Intell. Lab. Syst., 95, 188, (2009).

21. O. Ivanciuc, Internet Electron. J. Mol. Des. 3, 802, (2004).

22. J. Bi, K. P. Bennett, M. Embrechts, C. M. Breneman, M. Song, J. Mach. Learn. Res. 3, 1229, (2003).

23. J.-P. Doucet, F. Barbault, H. Xia, A. Panaye, B. Fan, Curr. Comp. Aid. Drug Des. 3, 263, (2007).

24. B. Schölkopf, A. J. Smola, R. C. Williamson, P. L. Bartlett, Neural Comput. 12, 1207, (2000).

25. C. C. Chang, C. J. Lin, LIBSVM: A library for support vector machines, March 6, 2010 (http://www.csie.ntu.edu.tw/ cjlin).

26. K. Tuppurainen, S. Lötjönen, R. Laatikainen, T. Vartiainen, U. Maran, M.Strandberg, T. Tamm, Mutat. Res., 247, 97, (1991).

27. D. F. V. Lewis, C. Ioannides, D. V. Parke, Xenobiotica 24, 401, (1994)

28. Z. Zhou, R. G. Parr, J. Am. Chem. Soc. 112, 5720, (1990). 